\title{
Classical wave propagation in strongly scattering media
}

\author{
J.H. Page ${ }^{\mathrm{a}, *}$, H.P. Schriemer ${ }^{\mathrm{a}}$, I.P Jones ${ }^{\mathrm{a}}$, Ping Sheng ${ }^{\mathrm{b}}$, D.A. Weitz ${ }^{\mathrm{c}}$ \\ a Department of Physics, University of Manitoba, Winnipeg, MB, Canada R3T 2N2 \\ b Department of Physics, Hong Kong University of Science and Technology, Clear Water Bay, \\ Kowloon, Hong Kong \\ ' Department of Physics and Astronomy, University of Pennsylvania, $209 \mathrm{~S} 33 \mathrm{rd}$ St., Philadelphia, \\ PA. 19104-6396, USA
}

\begin{abstract}
The transport of classical waves in strongly scattering media is investigated using ultrasonic techniques, allowing us to measure both the ballistic and scattered components of the wave field. We find that the ballistic propagation is dramatically slowed down by scattering resonances, although the group velocity remains well-defined. The propagation of the scattered waves is also strongly affected by resonant scattering, and is shown to be well described by using the diffusion approximation. A model based on the generalized coherent potential approximation gives a quantitative explanation of the experimental data.
\end{abstract}

PACS: 43.35.+d; 43.30. $+\mathrm{g} ; 62.30 .+\mathrm{d}$

Keywords: Wave propagation; Inhomogeneous media; Group velocity; Multiple scattering; Diffusion of sound

\section{Introduction}

Over the last decade, there has been renewed interest in the study of the multiple scattering of classical waves (light and sound) in strongly scattering media [1,2]. One reason for this interest is the realization that wave phenomena in strongly disordered inhomogeneous materials often exhibit radically different behaviour from that normally associated with waves. For example, the fact that wave propagation under the conditions of strong multiple scattering can be well described by using the diffusion approximation is quite remarkable, as the diffusion approximation completely ignores wave interference which is one of the most basic properties of waves. Even more remarkable is the complete change in the basic wave character implied by the possibility that a wave

\footnotetext{
${ }^{*}$ Corresponding author. Tel.: (204) 474-8752; fax: (204) 261-8489; e-mail: jhpage@cc.umanitoba.ca.
} 
may become localized in random and partially ordered materials. These and other related questions have motivated extensive research activity [1], although from the experimental point of view, most studies have involved only electromagnetic waves until quite recently.

In this paper, we summarize some of the recent achievements using sound to gain new information on the propagation of classical waves in strongly scattering materials $[3,4]$. By focusing on acoustic waves, we are able to exploit the ability of ultrasonic techniques to detect directly the wave field, rather than its intensity, allowing both phase and amplitude information to be measured. We have used this approach to study wave propagation in a simple disordered medium consisting of glass beads randomly closepacked in water; in the intermediate frequency range where the ultrasonic wavelength is comparable to the bead size, the large acoustic mismatch between glass and water ensures very strong scattering. Although the detected signals are dominated by multiply scattered waves, the phase sensitivity of ultrasonic transducers allows us to extract the weak signal that propagates ballistically through the medium without scattering out of the forward direction. This ballistic signal remains coherent to the incident pulse even though it is strongly affected by the scattering resonances, which cause a remarkable slowing down of the phase and group velocities. These scattering resonances also dramatically affect the propagation of the scattered waves, whose energy transport is shown to be well described by using the diffusion approximation. We are able to obtain additional insights into the nature of classical wave propagation in this strongly scattering regime by comparing the velocities of the ballistic and diffusive waves, and also to explain our results using a new theoretical approach that correctly accounts for the coupling between resonant scatterers.

\section{Ballistic propagation}

In Fig. 1, we illustrate how the ballistic pulse is extracted from the total signal detected in our ultrasonic experiments. Fig. 1(a) shows typical examples of the acoustic waveforms transmitted through a thin slab-like sample of glass beads of $0.5 \mathrm{~mm}$ radius in water. The incident pulse was generated using a focusing transducer, giving an excellent approximation to a point source, and the transmitted signals were detected using a miniature hydrophone, allowing the fully transmitted waveforms to be measured accurately [4]. The three waveforms shown in Fig. 1(a) were taken by translating the sample sideways while holding the transducers fixed, thereby measuring the propagation through different ensembles of scatterers in the sample. For times greater than about $6 \mu \mathrm{s}$, the detected field fluctuates wildly in amplitude and phase from position-to-position, reflecting the spatial fluctuations (speckles) characteristic of scattered waves. However, at the leading edge of the waveforms, the detected signal shows only small variations between speckles, indicating the presence of a ballistic component that is coherent, both spatially and temporally, with the incident pulse. We extract this ballistic signal by averaging the field over at least 100 speckles; this procedure cancels 


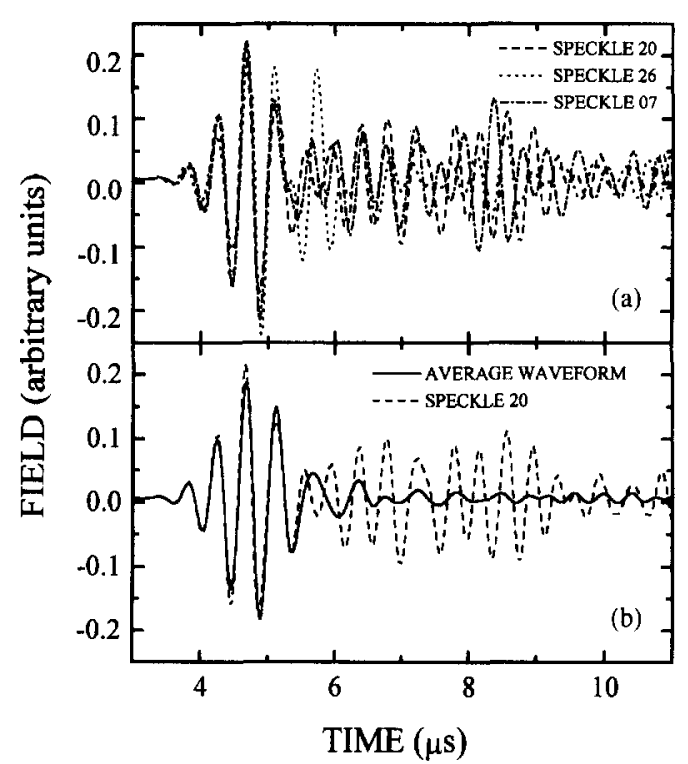

Fig. 1. (a) Time dependence of typical ultrasonic waveforms transmitted through a $3.4 \mathrm{~mm}$ thick sample containing glass beads of $0.5 \mathrm{~mm}$ radius in water. The sample thickness corresponds to about 4 mean free paths at the central frequency $(2.5 \mathrm{MHz})$ of the short incident pulse used in these measurements. (b) Average transmitted field, corresponding to the ballistic pulse (solid curve), is compared with the field in one of the speckles shown in the upper panel.

the scattered component because of its random phase fluctuations from speckle-tospeckle, giving the average waveform shown by the solid line in Fig. 1(b). In practice, the elimination of the scattered component from the ballistic pulse is facilitated by replacing the hydrophone by a large-diameter piezoelectric transducer, in which the spatial averaging is largely accomplished within the phase-sensitive detector itself.

The ballistic pulses for samples of different thicknesses are compared with the incident pulse in Fig. 2. Clear evidence of the dispersion is seen, both by the broadening of the transmitted pulses and by the differences in the propagation times of the wave crests (which travel at the phase velocity, $v_{p}$ ) and the pulse envelopes (which travel at the group velocity, $v_{g}$ ). However, despite the very strong resonant scattering (the mean free path is comparable to the ultrasonic wavelength throughout this frequency range), the locations of the peaks in the pulse envelopes, and hence the group velocity itself, remain well-defined. To measure accurately the frequency dependence of the group and phase velocities, we use a longer incident pulse consisting of about 10 oscillations and digitally filter the waveforms to form Gaussian pulses with relatively narrow frequency bandwidths. Over the dimensionless frequency range $1<k_{w} a<10\left(k_{w}\right.$ is the wave vector in water and $a$ is the bead radius), we find a very large variation, more than a factor of 2, in the group velocity (Fig. 3(a)), directly showing the dramatic effects of scattering on the propagation of the coherent, unscattered component. Even more striking are the extremely low values of the group velocities that are observed over 


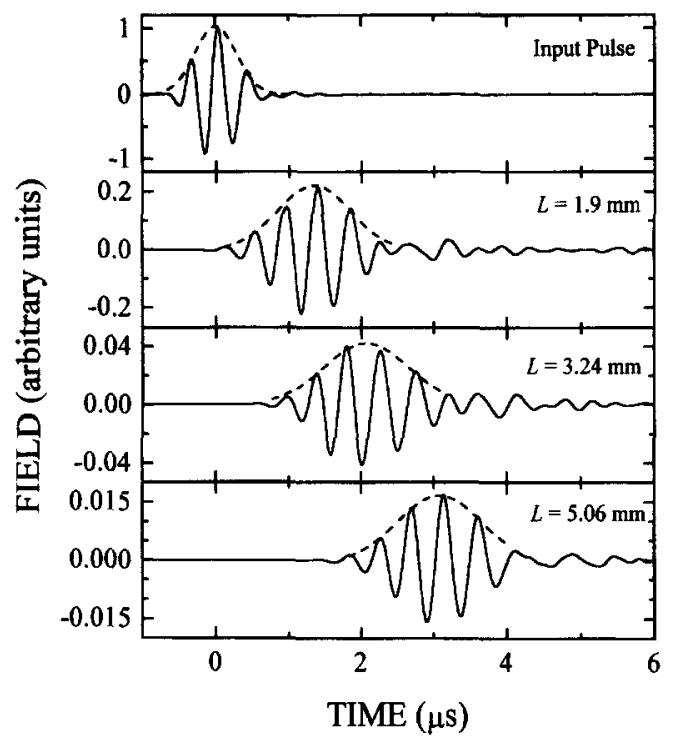

Fig. 2. Ballistic pulses transmitted through samples of different thicknesses (lower three panels). The input pulse is shown in the top panel.

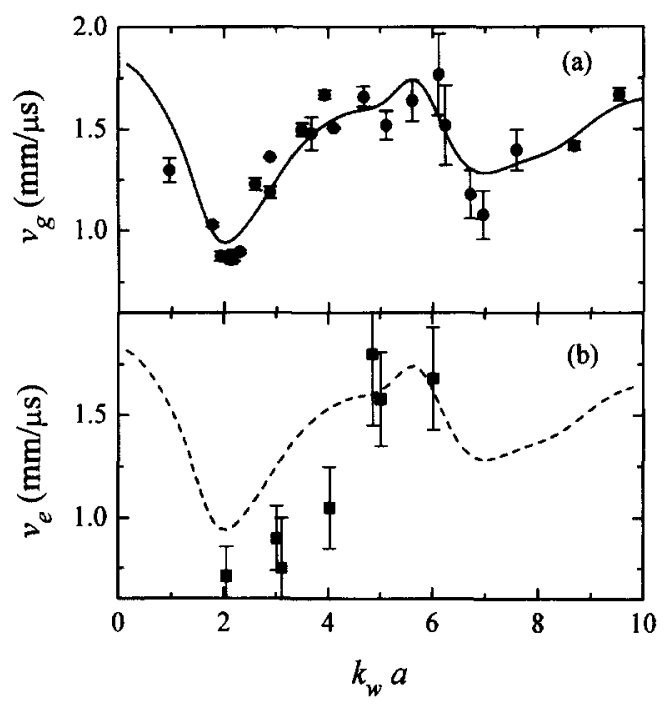

Fig. 3. (a) Frequency dependence of the group velocity. The solid symbols represent the experimental data which are compared with the predictions of the GCPA model (solid curve). (b) The energy velocity obtained from the measurements of the diffusion coefficient and transport mean free path for multiply scattered sound. The dashed curve is identical to the theoretical curve in (a) to facilitate comparison of the two velocities.

most of this frequency range, these being substantially less than the sound velocities in either glass or water.

The origin of these very slow velocities can be understood using a model based on the generalized coherent potential approximation (GCPA) [5,6], which overcomes 
a fundamental limitation of the traditional CPA approach to wave propagation in the intermediate frequency regime. To calculate the scattering quantitatively, we model a typical scatterer as an elastic sphere (glass) that is coated with a layer of water and embedded in a homogeneous effective medium whose properties account for the presence of the other scatterers in the system. The dispersion relation of acoustic waves in the medium is then determined by identifying the peaks of the spectral function, given by the negative imaginary part of the Green's function, in the frequency-wave vector plane [3]. A contour plot of the spectral function for our glass bead in water system is shown in Fig. 4, where the dispersion relation given by the peaks is highlighted by the solid line. Excellent agreement is found with our experimental data (solid symbols) which are determined from measurements of the phase velocity [3]. By numerically differentiating the theoretical dispersion curve, we calculate the group velocity and again find extremely good agreement with our data (Fig. 3(a)). The physical mechanism underlying the remarkable slowing down of the phase and group velocities can be understood as follows: because of the strong coupling between the resonant scatterers, the uniform effective medium sensed by the coherent ballistic propagation is very strongly renormalized, in much the same way as the quantum mechanical resonances are shifted when there is strong coupling between them. Thus, the ballistic pulse is still able to propagate coherently while being very strongly affected by the scatterers. Furthermore, we find, both experimentally and theoretically, that the group velocity does not lose its meaning despite the very strong scattering [3], thereby resolving a long-standing problem, first recognized by Sommerfeld [7] and Brillouin [8] and more recently discussed by van Albada et al. [9], in the definition of the group velocity in strongly scattering media.

\section{Diffusive propagation}

We next turn to the propagation of the scattered acoustic waves, which are responsible for most of the energy transport through strongly scattering materials. Although it is difficult to describe the multiple scattering of classical waves from first principles, considerable progress has been achieved through the use of the diffusion approximation, in which the transport of the configurationally averaged wave intensity is treated as a random-walk process. All phase information in the multiply scattered waves is neglected, and the propagation is characterized by the diffusion coefficient, $D=v_{e} l^{*} / 3$, where $v_{e}$ is the average velocity at which energy is transported and $l^{*}$, the transport mean free path, is the length over which the direction of propagation is randomized. Despite the success of this simple model in describing the propagation of light and microwaves in random media [1], it is only relatively recently that the application of the diffusion approximation to multiply scattered sound has been convincingly demonstrated [4].

We used pulsed experiments to measure the diffusion coefficient, $D$, from the temporal evolution of the transmitted acoustic intensity, which we determined by ensemble 


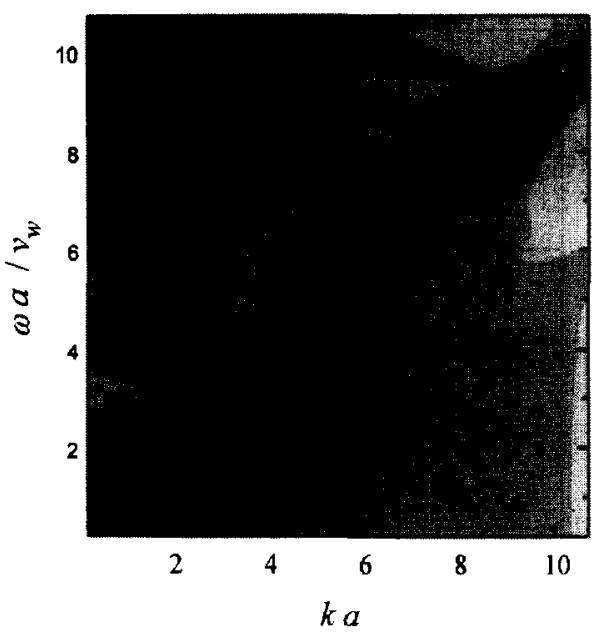

Fig. 4. Gray-scale contour plot, with dark being high and light being low, of the frequency and wave vector dependence of the spectral function. The peaks delineate the dispersion relation, shown by the solid black and white line. The open circles are experimental data, determined from the frequency dependence of the phase velocity. The dispersion relation for water is shown by the dashed line for comparison.

averaging the square of the envelope of the scattered sound field over a large number of independent speckles. Typical results are shown in Fig. 5, where we compare the diffusive time profiles measured at four different frequencies; for ease of comparison, the time profiles at each frequency are displaced vertically by equal amounts. A square input pulse of approximately $4 \mu \mathrm{s}$ was used for these measurements, ensuring a well defined ultrasonic frequency. The symbols represent the experimental data, and the solid lines are fits to the solution of the diffusion equation in which we have correctly accounted for the incident pulse width, the effects of absorption, reflections at the boundaries, and the location inside the sample of the effective diffusive source [4]. Excellent agreement between theory and experiment is shown in this figure for all the frequencies, demonstrating the validity of the diffusion approximation for multiply scattered sound [4] and allowing reliable values of the diffusion coefficient to be determined. Fig. 5 also shows that there is a large variation in the diffusive time profile with frequency, indicating that both the diffusion coefficient, $D$, and the absorption time, $\tau_{a}$, exhibit a strong frequency dependence.

In Fig. 6, we summarize the frequency dependence of $D$ and $\tau_{a}^{-1}$, measured for two different bead radii, $a=0.5$ and $0.25 \mathrm{~mm}$. The diffusion coefficient exhibits a strong dependence on both frequency and bead size. By contrast, the absorption rate exhibits a roughly linear increase with frequency but is independent of $a$. Thus, the absorption cannot be due to the usual viscous losses encountered in a fluid near a fluid-solid interface, since this mechanism gives an absorption rate proportional to both the surface area and the square root of frequency [10]. Instead, it appears that the absorption is intrinsic to the glass beads themselves, as the measured absorption depends solely on the amount of glass in the suspension and has a frequency-dependence characteristic of internal friction in glassy solids. 


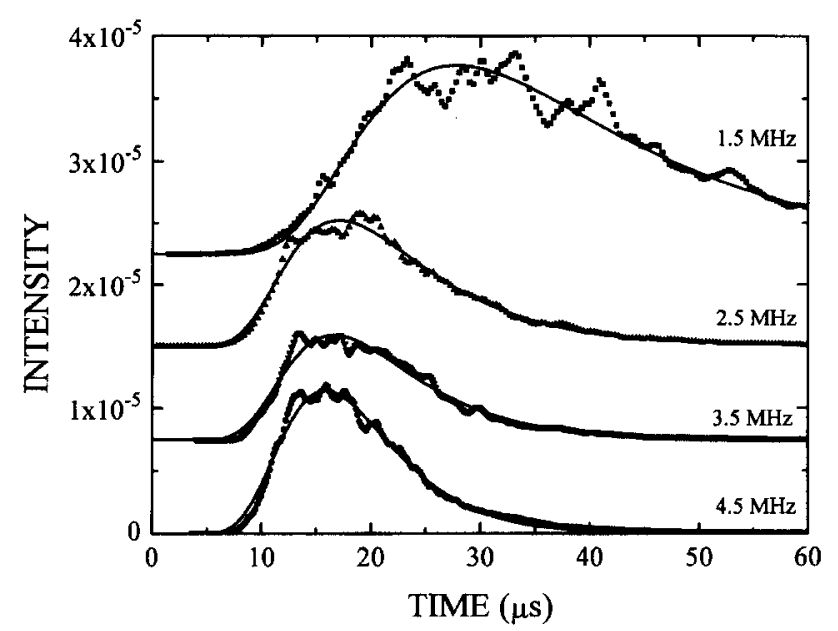

Fig. 5. Time profiles of the ensemble-averaged transmitted intensity of multiply scattered sound at the four incident frequencies shown. The experimental data (symbols) are compared with fits to diffusion theory (solid curves).

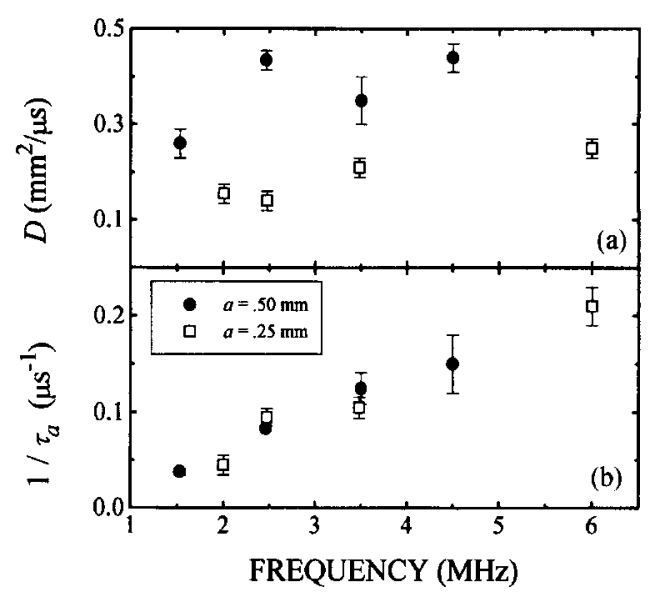

Fig. 6. Frequency dependence of the diffusion coefficient, $D$, and the absorption rate, $\tau_{a}^{-1}$, for beads of two different radii, $a$.

Further insight into the behaviour of the diffusion coefficient is obtained by noting that the minima and maxima in $D$ for the two bead sizes occur at the same values of $k_{w} a$ as the minima and maxima seen in the group velocity (Fig. 3(a)). This suggests that the group and energy velocities may have a similar frequency dependence. To investigate this interesting possibility, we used quasi-continuous-wave experiments to measure the transport mean free path $l^{*}$ from the thickness dependence of the absolute transmitted intensity [4]. From these data and our measurements of $D$, we obtain the results for the energy velocity, $v_{e}=3 D / l^{*}$, shown in Fig. 3(b). Near the first minimum in the group velocity, where the excitation of internal modes within the glass spheres 
causes the scattering mean free path to become less than half the wavelength, the energy velocity is a little slower than the already very slow group velocity, while at the higher frequencies, where the ratio of mean free path to wavelength is larger, the two velocities are indistinguishable within experimental error. Thus, most of the dramatic slowing down of wave propagation due to scattering resonances is captured by the group velocity that describes coherent wave transport. This suggests that a unified picture of wave transport within the generalized coherent potential approximation may be possible, thereby elucidating the close connection shown in Fig. 3 between the group and energy velocities which describe the transport of energy by the ballistic, and diffusive waves, respectively [11].

\section{Conclusions}

In this paper, we have focused on some new developments in the understanding of the classical wave propagation in multiply scattering materials that have been made possible using sound rather than light as the experimental probe: the measurement and definition of the group velocity under conditions of strong resonant scattering, and the relationship between the very slow group and energy velocities. Our measurements of multiply scattered sound may also have important applications in the non-destructive ultrasonic evaluation of very inhomogeneous materials, where we have recently demonstrated the feasibility of measuring the dynamics of the scatterers using a technique called diffusing acoustic wave spectroscopy [12].

\section{Acknowledgements}

We thank K.S. Beaty, M.L. Cowan and Xiaodun Jing for their assistance, and the Natural Sciences and Engineering Research Council of Canada and the North Atlantic Treaty Organization for supporting this project.

\section{References}

[1] P. Sheng (ed.), Scattering and Localization of Classical Waves in Random Media (World Scientific, Singapore, 1990).

[2] P. Sheng, Introduction to Wave Scattering, Localization and Mesoscopic Phenomena (Academic Press, San Diego, 1995).

[3] J.H. Page, P. Sheng, H.P. Schriemer, I. Jones, X. Jing and D.A. Wietz, Science 271 (1996) 634.

[4] J.H. Page, H.P. Schriemer, A.E. Bailey and D.A. Weitz, Phys. Rev. E 52 (1995) 3106.

[5] X.D. Jing, P. Sheng and M.Y. Zhou, Phys. Rev. A 46 (1992) 6513.

[6] P. Sheng, X. Jing and M. Zhou, Physica A 207 (1994) 37.

[7] A. Sommerfeld, Ann. Phys. 44 (1914) 177.

[8] L. Brillouin, Wave Propagation and Group Velocity (Academic Press, New York, 1960).

[9] M.P. van Albada, B.A. van Tiggelen, A. Lagendijk and A. Tip, Phys. Rev. Lett. 66 (1991) 3132.

[10] L.D. Landau and E.M. Lifshitz, Fluid Mechanics (Pergamon, Oxford, 1959).

[11] H.P. Schriemer, J.H. Page, P. Sheng and D.A. Weitz, unpublished.

[12] I.P. Jones, J.H. Page and D.A. Weitz, Phys. in Canada 52 (3) (1996) 129. 